\section{Utilidad del método de Carrea para estimar la estatura real en individuos peruanos}

\section{Carrea's method usefulness to estimate a peruvian population actual stature}

\section{Artículo Original}

Marcia Alejandra Moreno-Sánchez ${ }^{1, a}$, Hugo Humberto Caballero-Cornejo ${ }^{2, b}$, Carlos Alberto Suárez-Canlla ${ }^{2, c}$

${ }^{1}$ Universidad Científica del Sur, Facultad de Estomatología, Lima, Perú.

${ }^{2}$ Universidad Nacional Mayor de San Marcos, Facultad de Odontología, Lima, Perú.

a Cirujano Dentista.

b Doctor en Educación.

c Doctor en Estomatología.

\section{Correspondencia:}

Carlos Alberto Suárez Canlla: csuarezc@unmsm.edu.pe Facultad de Odontología de la Universidad Nacional Mayor de San Marcos, Calle Germán Amezaga №375, Facultad de Odontología, Ciudad Universitaria, Lima 1, Cercado de Lima, Lima-Perú

ORCID: 0000-0003-4719-1409

\section{Coautores:}

Marcia Alejandra Moreno-Sánchez: marcia.alejandra.ms@ gmail.com

ORCID: 0000-0002-1923-3686

Hugo Humberto Caballero-Cornejo: hcaballeroc@unmsm. edu.pe

ORCID: 0000-0002-7901-8523

\section{Editor invitado:}

Guido Marañón Vásquez

Universidade Federal do Rio de Janeiro, Brasil.

Objetivo. Determinar la utilidad del método de Carrea para la estimación de la talla o estatura. Métodos. Enfoque cuantitativo de diseño observacional y trasversal. La muestra ascendió a 188 estudiantes (84 mujeres, 104 varones) con sus respectivos modelos de estudio cuya edad promedio fue 21,07 años. Se determinó la estatura real y estimada por medio del tallímetro y el método Carrea respectivamente para establecer la diferencia absoluta entre la talla real y estimada con el fin de determinar - por medio del estadístico $t$ de Student para muestras independientes - si esta diferencia resultaba significativa. El coeficiente de correlación intraclase fue utilizado para determinar la fuerza de concordancia entre la talla real y estimada. Resultados. La diferencia entre el promedio de la estatura real y el promedio de la estatura estimada fue de 4,4 centímetros. La diferencia absoluta promedio entre la estatura real y la estatura estimada fue de 6,9 centímetros. El estadístico t de Student para muestras independientes encontró que la estatura estimada difiere significativamente de la estatura real. La fuerza de concordancia entre la estatura real y la estatura estimada fue de 0,791 (ICC $=0,791$ ) ello permitió categorizar a dicha concordancia como buena. Conclusiones. El método de Carrea resulta útil en la estimación de la estatura. Sus limitaciones en términos de exactitud deben tomarse en consideración antes de emitir una conclusión, especialmente si los individuos analizados guardan relación con contextos forenses en los que la identificación juega un papel relevante en el ámbito jurídico y humanitario.

Palabras clave: Odontología forense; Antropometría; Estatura (Fuente: DeCS BIREME).

\section{Abstract}

Objective. To determine the usefulness of Carrea's method for estimating the stature. Methods. This is a quantitative observational and cross-sectional design study. The used sample was of 188 students (84 women, 104 men) 21.07 year old average and their respective plaster models. The actual stature and estimated stature for each student was determined by means of the height rod and the Carrea's method respectively to establish the absolute difference between them and to determine - by means of the Student's $t$ test for
Conflicto de intereses: los autores declaran no tener conflictos de interés.

Fuente de financiamiento: ninguno.

Recibido: $13 / 05 / 20$

Aceptado: $04 / 08 / 20$

Publicado: $16 / 11 / 20$ 
independent samples - if this difference was significant. The intraclass correlation coefficient was used to determine the concordance strength between the actual and estimated stature. Results. The difference between actual stature average and estimated one was 4.4 centimeters. The absolute average difference between them was 6.9 centimeters. These differences were significant to Student's t test for independent samples. The concordance strength between the real and estimated stature was 0.791 (ICC $=0.791$ ), which allowed us to categorize this concordance as good. Conclusions. Carrea's method is useful in stature estimation. Its limitations in terms of accuracy must be taken into consideration before reaching a conclusion, especially if the individuals analyzed are related to forensic contexts in which identification plays a relevant role in the legal and humanitarian field.

Keywords: Forensic dentistry; Anthropometry; Body height (Source: MeSH NLM).

\section{Introducción}

Para lograr la identificación de restos humanos esqueletizados y cadáveres en avanzado estado de putrefacción los peritos forenses deben determinar su perfil biológico, es decir; estimar su estatura, edad, sexo y raza. Este perfil biológico en conjunto con las características individualizantes - odontograma, patologías, lesiones antemortem etc. - constituyen la data postmortem que se comparará posteriormente con la data antemortem de la presunta persona desaparecida. El resultado de dicha comparación arribará a la identificación del cadáver, sin embargo, en otras ocasiones ello no es posible ${ }^{1,2,3}$.

El perito forense debe conocer el contexto en el cual se encuentra el(los) cadáver(es) o resto(s) esqueletizado(s) sin identidad antes de brindar una conclusión respecto de su identificación. En este sentido, existen poblaciones abiertas, cerradas y mixtas; la identificación de individuos dentro de una población cerrada (muertes colectivas donde se tiene información precisa sobre el número y presuntas identidades de las víctimas) ${ }^{4}$ resulta más sencilla respecto de una población abierta. En estos contextos cerrados; existen ciertos casos forenses en los cuales los restos esqueletizados presentan diferencias importantes entre sus tallas. Así, la estimación de la estatura cobra vital importancia porque saca a relucir estas diferencias que orientan decididamente el proceso de identificación.

En otros contextos es común hallar restos humanos esqueletizados en mal estado de conservación - debido a múltiples factores tafonómicos - que deterioran el tejido óseo ${ }^{5,6}$, entre ellos los huesos largos, los cuales son útiles para la estimación de la estatura. Para estos casos, la estimación de la talla a través de los dientes resulta un recurso importante para determinar el perfil biológico de los restos que, dentro de un contexto cerrado, marcaría una pauta importante para su identificación.

La fórmula para estimar la estatura a través de los dientes la sustentó el Dr. Ubaldo Carrea en la Universidad de Buenos Aires - Argentina (1920). Esta fórmula establece el cálculo de la talla a partir del arco y radio-cuerda, los mismos que están determinados por las medidas hechas al incisivo central, lateral y canino inferiores. A partir del arco se calcula la talla máxima y a partir del radio-cuerda la talla mínima ${ }^{7}$.
Existen investigaciones con distintos enfoques en distintas partes del mundo respecto al método de Carrea para aplicarlo de diferentes maneras, por ejemplo, Garrido et al. ${ }^{8}$ realizaron pruebas para obtener una versión en la que consideraban la medición de los dientes deciduos superiores en lugar de los permanentes inferiores como indicaba Carrea. En Brasil, De Almeida ${ }^{9}$ comparó el método de Carrea con el índice cefalométrico de Retzius y el índice facial. Lima et al. ${ }^{10}$ aplicaron el método de Carrea en arcos dentales normales con apińamiento y con diastemas para probar su aplicabilidad. En el Perú también se han desarrollado investigaciones respecto a la tesis de Carrea para demostrar su aplicabilidad en la población peruana y, al igual que éstas, la presente investigación pretende validar el método de Carrea para poder aplicarlo en los distintos casos forenses que guardan relación con la identificación de restos humanos sin identidad que están inmersos en distintos contextos forenses como feminicidios, muertes extrajudiciales, violación a los derechos humanos, muertes en contextos de desastres masivos y otros.

Por todo esto, el objetivo de este estudio fue determinar la utilidad del método de Carrea - a través de la diferencia entre la talla real y estimada - para su validación y posterior uso en la población peruana.

\section{Métodos}

El enfoque de esta investigación es cuantitativo, el diseño es observacional y trasversal, y enmarca dentro de la odontología forense.

La población estuvo constituida por los 371 estudiantes matriculados en el periodo académico 2013-2 de la Facultad de Odontología de la Universidad Nacional Mayor de San Marcos (UNMSM). La muestra de estudio estuvo integrada por 180 estudiantes, los cuales fueron seleccionados por conveniencia de acuerdo a los criterios de selección. La unidad de análisis estuvo constituida por los modelos de yeso de la arcada dental mandibular.

Los siguientes criterios de inclusión fueron considerados para seleccionar la muestra: (a) estudiantes con aparente buen estado de salud física y (b) estudiantes que cuenten con sus incisivos y caninos mandibulares. Los criterios de exclusión: (a) estudiantes con patologías o deformaciones evidentes en su columna vertebral y extremidades inferiores producto de alteraciones en su crecimiento y 
desarrollo; así como accidentes que produzcan alteraciones importantes en la columna y miembros inferiores, (b) incisivos y caninos que presenten patologías relacionadas a alteraciones de tamaño, número, erupción, forma y estructura del tejido dental, (c) incisivos y caninos que presenten alteraciones de posición dentro de la arcada dentaria: giroversiones, migraciones etc., (d) incisivos y caninos que presenten tratamientos rehabilitadores como coronas, carillas y/o restauraciones que alteren el tamaño de la corona.

Luego de contar con la muestra de estudio se asignó un código numérico a todos y cada uno de los estudiantes, el mismo que reemplazó sus apellidos y nombres con la finalidad de mantener en reserva su identidad. Además, se obtuvo el consentimiento informado de cada uno de ellos - a quienes se les indicó el objetivo de la investigación - y se mantuvo la estricta privacidad y confidencialidad de sus datos por parte del investigador responsable, quién fue la única persona que realizó la recolección de los datos con acceso a sus nombres y apellidos. La Facultad de Odontología de la UNMSM aprobó la ejecución de los pasos detallados anteriormente.

Posteriormente se procedió a determinar la estatura real y la estatura estimada de todos los integrantes de la muestra.

Para determinar la estatura real de las personas se utilizó un tallímetro de madera. A todos se les solicitó que estuvieran sin zapatos, con la espalda recta y la cabeza con el plano de Frank-Fort paralelo al piso, para mayor precisión se usó una escuadra para proyectar el vertex al tallímetro.

Para hallar la estatura estimada se tomó una impresión del arco dentario inferior. Se utilizaron cubetas cribadas, de acuerdo al tamaño del arco mandibular, y el alginato como material de impresión. Posteriormente se hizo el vaciado empleando yeso piedra (yeso piedra tipo 3 cuya dosificación agua/polvo fue de $30 \mathrm{ml} / 100 \mathrm{~g}$, con un tiempo de retiro del modelo de 45 minutos, de una expansión de $0,04 \%$ y una resistencia a la compresión de $632 \mathrm{~kg} / \mathrm{cm}^{2}$ ). El modelo de estudio obtenido fue debidamente rotulado con el código numérico correspondiente a la persona a la cual ya se le había determinado su talla real.

En cada uno de los modelos se halló la longitud del diámetro mesio-distal de los incisivos central y lateral, y del canino de una misma hemiarcada mandibular. Las medidas fueron obtenidas a través de un vernier digital previamente calibrado para expresar dichas medidas en milímetros. El instrumento utilizado tuvo un rango de medida entre 0 a $150 \mathrm{~mm}$, con una exactitud de 0,03 $\mathrm{mm}$, cuya unidad de medida puede pasar de pulgadas a milímetros y viceversa, y una pantalla con un interfaz de salida a una computadora. La decisión respecto a que hemiarcada utilizar - derecha o izquierda - para realizar las mediciones tuvo en consideración los criterios de inclusión y exclusión ya mencionados. Luego, se realizó la sumatoria de los tres diámetros mesio-distales para obtener el primer parámetro de la fórmula de Carrea: el arco. Asimismo, se obtuvo la distancia lineal desde la superficie mesial del incisivo central inferior hasta la superficie distal del canino inferior, con dicha longitud se obtuvo el segundo parámetro de la fórmula de Carrea: el radio-cuerda. Inmediatamente después se utilizaron las fórmulas correspondientes para establecer la estatura máxima y mínima que guardan relación con el arco y radio-cuerda antes mencionados.

Estatura máxima $=\underline{\operatorname{arco}(\mathrm{mm}) \times 6 \times 3,1416 \times 10}$

Estatura mínima $=\underline{\text { radio-cuerda }(\mathrm{mm}) \times 6 \times 3,1416 \times 10}$

Respecto al uso de las fórmulas, y tal como aparece en la tesis de Carrea - quién es el autor del método -, la talla masculina guarda relación o es igual a la estatura máxima y la femenina es igual a la estatura mínima.

Todas las mediciones y los resultados de la aplicación de las fórmulas fueron anotados en las fichas de recolección de datos y dentro de la tabla matriz o base de datos diseñada previamente para compilar toda esta información.

El procesamiento y análisis estadístico de la información se realizó a través del programa estadístico SPSS versión 21. El análisis univariado comprendió la descripción de la estatura real, la estatura estimada y la diferencia absoluta entre ellas por medio de medidas de resumen (valor mínimo, máximo, media y desviación estándar) para el total de la muestra clasificadas por sexo.

Para determinar la concordancia entre las dos estaturas real y estimada - se calculó el índice de correlación intraclase con el respectivo gráfico de dispersión de puntos.

Se aplicó la prueba t de Student para muestras independientes para comparar las medias de las dos estaturas (real y estimada) y determinar si existen diferencias entre ellas. Previamente a todo esto se verificó que los datos presenten distribución normal aplicando la prueba de Kolmogorov-Smirnov. Las pruebas fueron trabajadas a un nivel de significancia de $5 \%$.

\section{Resultados}

Los integrantes de la muestra se distribuyeron por edad y sexo respectivamente, en dicha distribución resalta el mayor número de hombres $(55,3 \%)$ en relación a las mujeres $(44,7 \%)$. La edad promedio de los varones y mujeres fue de 20,49 y 21,54 años respectivamente (Tabla 1 ).

La media y las medidas de dispersión respecto del arco y radio-cuerda del método de Carrea - de todos y cada uno de los estudiantes - tuvieron los siguientes resultados: la longitud promedio del radio-cuerda fue $17,01 \mathrm{~mm}$ y la longitud promedio del arco fue $18,55 \mathrm{~mm}$ (Tabla 2).

Los valores que se presentan en la tabla 3 se expresan en centímetros. La tabla muestra el promedio de la estatura real y el promedio de la estatura estimada (medias). Al comparar el promedio de la estatura real con el promedio de la estatura estimada - para el sexo femenino - se 
puede inferir que existe una sobreestimación de la estatura estimada respecto de la real en 3,5 centímetros. Igual comportamiento presentan los varones, es decir, hay una sobreestimación de la estatura estimada respecto de la real en 5,1 centímetros. Cuando se tomó la totalidad de la muestra, la sobreestimación de la estatura estimada respecto de la real se mantuvo: 4,4 centímetros.

Las diferencias entre la estatura real y estimada de todos y cada uno de los integrantes de la muestra de estudio tuvo como resultado una infraestimación y una sobreestimación de la estatura. La infraeestimación se presentó cuando la estatura real fue mayor que la estimada y la sobreestimación se presentó cuando la estatura estimada fue mayor que la real. Las diferencias entre la estatura real y estimada se presentaron como números positivos (infraestimación) y negativos (sobreestimación). A dichos números se les aplicó el artificio matemático denominado valor absoluto, es decir, establecer su valor numérico sin tener en cuenta su signo. Dicho procedimiento se realizó para hallar la diferencia absoluta promedio entre la estatura real y la estatura estimada, hecho que no se hubiese logrado sin eliminar los signos positivos y negativos que resultaron de la sustracción. Así, la diferencia absoluta promedio - tanto para mujeres y varones - fue de 6,3 y 7,4 centímetros respectivamente. Al tomar la totalidad de la muestra, la diferencia absoluta promedio entre la estatura real y estimada fue de 6,9 centímetros (Tabla 4).

Se indicó anteriormente que la diferencia entre los promedios totales de la estatura real y estimada fue de 4,4 centímetros; se indicó también que la diferencia absoluta entre los promedios totales de la estatura real

Tabla 1. Distribución porcentual de varones y mujeres de la muestra de estudio y sus edades promedio

\begin{tabular}{lcc}
\hline Variables & $\mathbf{n}$ & $\%$ \\
\hline Sexo & & \\
Femenino & 84 & 44,7 \\
Masculino & 104 & 55,3 \\
Total & 188 & 100 \\
Edad (media y DE*) & & \\
Femenino & & $20,49 \pm 2,9$ \\
Masculino & & \\
Total & & $21,54 \pm 3,4$ \\
\hline
\end{tabular}

* DE= Desviación estándar

Tabla 2. Distribución de las personas por sexo, según el parámetro de Carrea

\begin{tabular}{lcccccc}
\hline Sexo & Parámetro & $\mathbf{n}$ & Mínimo & Máximo & Media & DE $^{*}$ \\
\hline Femenino & Radio-cuerda $(\mathrm{mm})$ & 84 & 14,66 & 19 & 17,01 & 0,82 \\
Masculino & Arco $(\mathrm{mm})$ & 104 & 16,77 & 21,39 & 18,55 & 0,91 \\
\hline
\end{tabular}

* DE= Desviación estándar

Tabla 3. Distribución de la estatura real y estimada de las personas según el sexo

\begin{tabular}{lccccc}
\hline Sexo & $\mathbf{n}$ & Mínimo & Máximo & Media & DE $^{*}$ \\
\hline Femenino & \multirow{2}{*}{$\begin{array}{c}\text { Real } \\
\text { Estimada }\end{array}$} & 139,9 & 169,2 & 156,8 & 6,1 \\
Masculino & & 138,2 & 179,1 & 160,3 & 7,7 \\
$\quad$ Real & 104 & 157,8 & 185,0 & 169,8 & 5,3 \\
$\quad$ Estimado & & 158,1 & 201,6 & 174,9 & 8,6 \\
Total & & & & & \\
$\quad$ Real & 188 & 139,9 & 185,0 & 164,0 & 8,6 \\
$\quad$ Estimado & & 138,2 & 201,6 & 168,4 & 11,0 \\
\hline
\end{tabular}

* DE= Desviación estándar

Tabla 4. Diferencia absoluta entre la estatura real y la estatura estimada

\begin{tabular}{lccccc}
\hline Sexo & $\mathbf{N}$ & Mínimo & Máximo & Media & DE* $^{*}$ \\
\hline Femenino & 84 & 0,1 & 26,3 & 6,3 & 5,6 \\
Masculino & 104 & 0 & 34,5 & 7,4 & 6,7 \\
Total & 188 & 0 & 34,5 & 6,9 & 6,2 \\
\hline
\end{tabular}

*DE= Desviación estándar 
y estimada fue de 6,9 centímetros. Al comparar ambas diferencias $(4,4$ y 6,9 centímetros) se observa que hay mayor discrepancia entre la estatura real y la estatura estimada al utilizar la diferencia absoluta promedio.

Los datos antes detallados indicaron la necesidad de utilizar un estadístico inferencial para determinar si efectivamente existe diferencia significativa entre la talla real y estimada. Así, la prueba t de Student para muestras independientes fue utilizada para demostrarlo.

Los valores que se aprecian en la tabla 5 permiten inferir que efectivamente existe diferencia significativa entre la estatura real y estimada $(p<0,001)$. En otros términos; la estatura estimada difiere significativamente de la estatura real, hecho que permite - en términos estrictamente matemáticos - concluir respecto de la limitada precisión del método de Carrea para estimar la talla, pero en términos estrictamente forenses esta diferencia tiene otra connotación.

Se utilizó el índice de correlación intraclase (ICC) para medir la fuerza de concordancia entre la estatura real y la estatura estimada. El valor del índice obtenido fue de 0,791 . Este resultado permitió categorizar a la concordancia de la talla real con la estimada como buena $(0,71-0,90)$. Ello puede apreciarse en la figura que se presenta a continuación, la cual presenta la dispersión de puntos formada por las estaturas ya mencionadas.

\section{Discusión}

Los resultados de la investigación denotan su importancia y utilidad porque contribuirán decididamente en el proceso de identificación forense, más precisamente en aquellos casos forenses que se encuentran dentro de una población cerrada cuyos individuos sin identidad presentan discrepancias notables entre sus tallas. Así, el odontólogo forense utilizará estos resultados como una herramienta importante para la identificación de cadáveres y restos humanos esqueletizados que se encuentran en distintos contextos forenses como conflictos armados, muertes masivas, feminicidios, violación a los derechos humanos y muchos otros.

De otro lado, el presente estudio permitió conocer las fortalezas y limitaciones de las fórmulas de Carrea para estimar la estatura, las cuales deberán tenerse en consideración al momento de ser aplicadas a los distintos casos forenses, especialmente en aquellos casos que impliquen restos esqueletizados deteriorados o incompletos - debido a los distintos factores tafonómicos: ambientales, individuales, criminales y culturales - que han hecho mella en el tejido óseo ${ }^{6}$ y dejan al tejido dentario como último recurso para establecer la tétrada de la identificación. Al respecto; los resultados de esta investigación muestran que existe una diferencia de 6,9 centímetros entre la talla real y estimada, ello indica - a primera vista

Tabla 5. Comparación entre la estatura real y estimada

\begin{tabular}{|c|c|c|c|c|c|c|}
\hline \multirow{2}{*}{ Sexo } & \multirow[b]{2}{*}{$\mathbf{n}$} & \multicolumn{2}{|c|}{ Real } & \multicolumn{2}{|c|}{ Estimada } & \multirow{2}{*}{ Valor $p^{\dagger}$} \\
\hline & & Media & $\mathrm{DE}^{*}$ & Media & $\mathrm{DE}^{*}$ & \\
\hline Femenino & 84 & 156,8 & 6,1 & 160,3 & 7,7 & 0,001 \\
\hline Masculino & 104 & 169,8 & 5,3 & 174,9 & 8,6 & $<0,001$ \\
\hline Total & 188 & 164,0 & 8,6 & 168,4 & 11,0 & $<0,001$ \\
\hline
\end{tabular}

* DE= Desviación estándar

† Prueba t de Student para muestras independientes

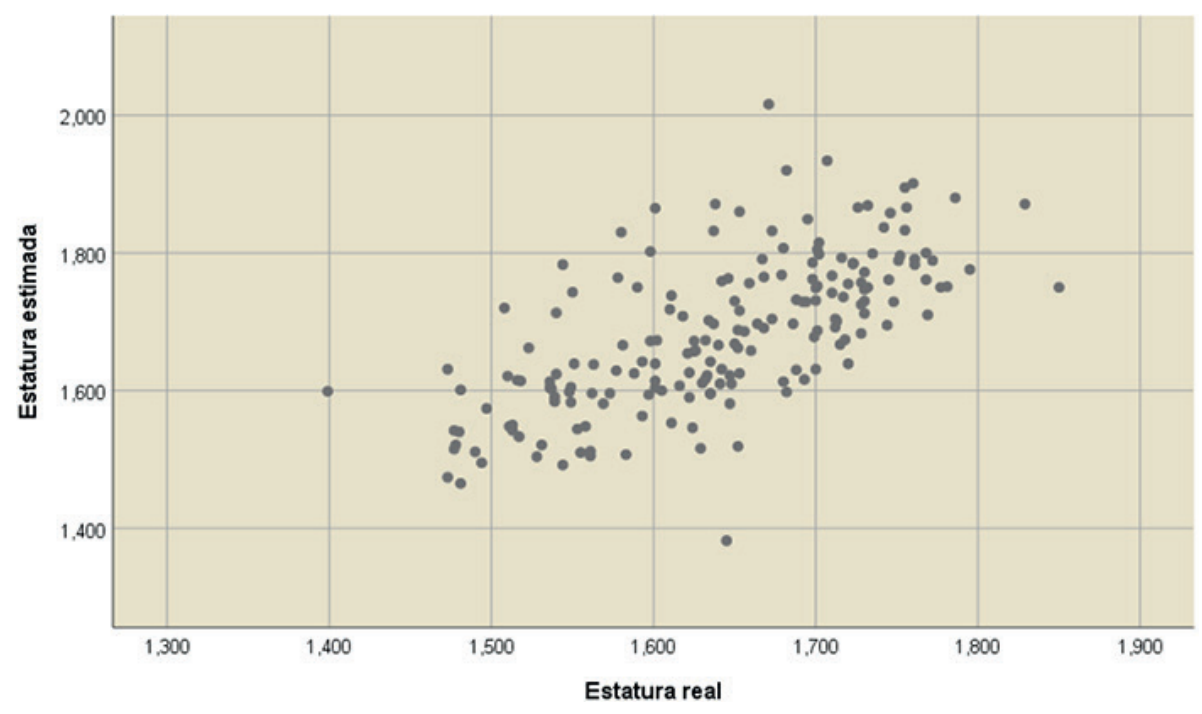

Figura. Imagen que grafica las coincidencias entre la estatura real y estimada

Fuente: datos del análisis estadístico (ICC) 
- la poca precisión del método de Carrea para estimar la estatura. Sin embargo, se debe destacar que los métodos de estimación de la estatura que utilizan la longitud de los huesos largos para estos fines, también brindan sus resultados con rangos bastante amplios; por ejemplo, el método desarrollado por Genoves (1.967) - que utiliza el fémur para calcular la talla - brinda un rango de 6,84 centímetros entre la talla mínima y máxima, dicho de otra manera, presenta una desviación estándar de 3,42 centímetros alrededor de la estatura calculada. Asimismo, el método de Genovés presenta un rango entre la talla mínima y máxima alrededor de los 5,62 centímetros cuando utiliza la tibia para predecir la estatura ${ }^{5,11,12}$. Es preciso señalar que no existe un método capaz de predecir con exactitud la estatura de una persona a partir de sus huesos o sus dientes y, al igual que los otros indicadores que conforman la tétrada de la identificación, siempre se presentarán rangos - amplios o reducidos - que el forense deberá tomar en consideración al momento de asignar el género y la raza a un esqueleto, así como al momento de calcular su edad y estatura 3-6,11.12. A esto se debe agregar que, si bien los métodos de estimación de la talla y la edad no ofrecen una precisión matemática en sus predicciones, sus diferencias respecto a la verdadera talla y edad no quitan su utilidad pues, el comportamiento de las variables biológicas en las que se fundamentan tampoco presentan dicho comportamiento matemático, en especial aquellas relacionadas a determinar la tétrada de la identificación: el crecimiento y desarrollo de los tejidos, la degeneración fisiológica de los mismos, sus dimensiones y sus características fisiológicas intrínsecas a ellos. En tal sentido, los hallazgos de este estudio exponen claramente las limitaciones - en términos de precisión - de la fórmula de Carrea al momento de aplicarla.

Per se a sus limitaciones, los resultados aquí obtenidos muestran las bondades del método de Carrea, las cuales pueden resumirse en su utilidad para predecir la estatura en aquellos casos en los que solo se cuenta con segmentos del maxilar y/o mandíbula.

Cavalcanti et al. ${ }^{13}$ demostraron que - utilizando el método de Carrea - la altura real del individuo coincidía con el intervalo entre las alturas mínima y máxima en un $36,0 \%$ para el lado derecho y un $48,0 \%$ para el lado izquierdo; mientras que utilizando el método modificado hubo un $96,0 \%$ de coincidencias para ambos lados. Por tanto, el método modificado fue más eficiente para estimar la estatura que el método primigenio de Carrea. El presente estudio no realizó comparaciones que lleven a coincidencias entre la altura real estimada, pero si utilizó el índice de correlación intraclase (ICC) para medir la fuerza de concordancia entre la estatura real y la estatura estimada, la misma que fue catalogada como buena (ICC $=0,791)$.

Lima et al. ${ }^{10}$ informan que la estatura es una característica medible del cuerpo y útil para la identificación humana, la cual puede incluir o excluir a un individuo de una lista de personas desaparecidas. Además, mencionan que, considerando una dentición normal y apińada, el índice de Carrea presento un porcentaje de éxito satisfactorio entre el 72,2\% y 95,2\%, sin diferencias estadísticamente significativas entre los sexos o entre los lados derecho e izquierdo. Mencionan también que la presencia de diastemas redujo el número de concordancias a un $62,5 \%$. Así, concluyen que el índice de Carrea es un método confiable para la estimación de la estatura en arcos dentarios normales y apiñados, así como útil en hombres y mujeres, y en los lados derecho e izquierdo, pero dicha eficiencia disminuye con la presencia de diastemas. Al respeto, se debe mencionar que la investigación aquí desarrollada considera también que el método de Carrea es útil para estimar la estatura, pero dicha utilidad debe tener en consideración las diferencias entre la estatura real y estimada que limitan su precisión, hecho que resulta importante cuando se aplica a los casos forenses. Este estudio también considera que la estatura puede incluir o excluir a un individuo de una lista de personas desaparecidas, siempre y cuando se trate de poblaciones cerradas en las cuales se tiene información precisa sobre el número y presuntas identidades de las víctimas, pero en poblaciones abiertas dicho procedimiento resulta contraproducente.

El presente estudio permite inferir que el método de Carrea es útil para predecir la estatura, y su exactitud mejoraría al utilizarla en conjunto con otros métodos de estimación de la estatura. En aquellos casos forenses en los que solo se cuenta con dientes para determinar la tétrada de la identificación su utilidad resultas más relevante aún. Sin embargo, el estudio de Rekhi et al. ${ }^{14}$, en el cual se evaluó la confiabilidad del índice de Carrea en una población india, se obtuvo como resultado un éxito del 94,03\% en la predicción de la estatura para los varones cuando la dentición fue normal y un éxito del $87,87 \%$ en la predicción de la estatura para las mujeres cuando la dentición estuvo apiñada. Por todo ello, Rekhi et al. concluyeron que el índice de Carrea es un método confiable para la estimación de la altura en arcos con denticiones normales y apiñados, así como útiles para ambos sexos. Luego de la lectura de este párrafo se desprenden algunas discrepancias entre la investigación ejecutada por Rekhi y la ejecutada aquí. Una de las diferencias más importantes corresponde a la conclusión de Rekhi : “... el indice de Carrea es un método confiable para la estimación de la altura ..." (en especial en el término confiable) pues, la investigación aquí desarrollada sí considera útil a la fórmula de Carrea, pero no confiable en todo el sentido de la palabra. Por el contrario, asume sus limitaciones las cuales ya fueron mencionadas anteriormente.

Rayapureddy et al. ${ }^{15}$ efectuaron un estudio con el objetivo de correlacionar las dimensiones de los dientes mandibulares anteriores con la estatura real utilizando el índice de Carrea - a través del índice de correlación de Pearson - para conocer la aplicabilidad y validez de este índice en una población india. La conclusión a la que llegan los investigadores es "Aunque se estableció una correlación entre la estatura real y la estatura estimada, el indice de Carrea como herramienta de predicción puede no proporcionar estimaciones precisas. Sin embargo, solo 
podria actuar como una herramienta auxiliar en la identificación de personas". Al respecto; el presente estudio no utilizó la correlación de Pearson para determinar la relación entre la estatura real y estimada, sino que optó por el índice de correlación intraclase que es capaz de ponderar proporcionalmente la variabilidad que le es inherente a toda medición. Dicha variabilidad puede dividirse en tres componentes: (a) la variabilidad debida a las diferencias entre los integrantes de la muestra, (b) la variabilidad debida a las diferencias entre los observadores o inherente a un mismo observador y (c) una variabilidad inexplicable asociada al error inherente a toda medición ${ }^{16}$. En este sentido; el presente estudio obtuvo un índice de correlación intraclase catalogado como bueno (ICC=0,791), el mismo que permite inferir que el método de Carrea resulta útil para estimar la estatura. De otro lado, el estudio realizado por Rayapureddy concluye que el índice de Carrea podría actuar como una herramienta auxiliar en la identificación de personas. Frente a este respecto; la presente investigación considera que la estimación de la estatura a través del índice de Carrea resulta útil en el proceso de identificación de cadáveres en mal estado de conservación y en restos humanos esqueletizados - incluidos dentro de una población cerrada - con diferencias importantes entre sus tallas.

En conclusión, el método de Carrea resulta útil en la estimación de la estatura. Sus limitaciones en términos de exactitud deben tomarse en consideración antes de emitir una conclusión, especialmente si los individuos analizados guardan relación con contextos forenses en los que la identificación juega un papel relevante en el ámbito jurídico y humanitario.

\section{Referencias bibliográficas}

1. Comité Internacional de la Cruz Roja. Personas desaparecidas, análisis forense de $\mathrm{ADN}$ e identificación de restos humanos. $2^{\circ} \mathrm{Ed}$. Ginebra: CICR Press; 2.010. Capítulo 1, Introducción a la identificación forense de restos humanos; p. 9-12.

2. Comité Internacional de la Cruz Roja. 1º Ed. Identificación forense de restos humanos. Ginebra: CICR Press; 2014. 12 p.

3. Ramey K. Manual de antropología forense. 1º Ed. Sánchez-Rodrigo C, traslator. Barcelona: Bellaterra editores; 2008. Capítulo 13, Análisis de laboratorio; 293-4.

4. Jiménez G. Manual para la identificación de cadáveres. Bogotá: Instituto Nacional de Medicina Legal y Ciencias Forenses. Capítulo 8, Manejo de información y documentos; p. 145-47.

5. Sanabria C. Antropología forense y la investigación médico legal de las muertes. $2^{\circ}$ ed. Bogotá: Asociación Colombiana de Antropología Forense; 2008. Capítulo V, Tafonomía forense; p. 175-212.
6. Fondebrider L, De Mendoca M. Protocolo modelo para la investigación forense de muertes sospechosas de haberse producido por violación de los derechos humanos. $1^{\text {a }}$ Ed. México: Oficina de alto comisionado para los derechos humanos de las Naciones Unidas; 2001. 50 p.

7. Carrea U. Ensayos odontométricos [tesis doctoral]. [Buenos Aires]: Facultad de Ciencias Médicas, Universidad Nacional de Buenos Aires; 1920. 82 p.

8. Garrido Y, Zavando D, Suazo I. Estimación de la estatura a partir de las dimensiones de la dentición temporal. Int J Odontostomat [Internet] 2012 [citado el 15 de mayo de 2013]; 6(2):139-43. Disponible en: https://scielo.conicyt.cl/pdf/ijodontos/v6n2/art04.pdf. DOI:10.4067/ S0718-381X2012000200004.

9. De Almeida C. Avaliaçao do índice de Carrea comparado ao índice cefalométrico de Retzius e índice facial [tesis de maestría]. [Sao Paulo]: Facultad de Odontología, Universidad Estatal de Campinas; 1995. 157 p.

10. Lima L, Da Costa R, Tinoco P, Rabello Daruge Junoir E. Stature Estimation by Carrea's index and its reliability in different types of dental alignment. J Forensic Odontostomatol [Internet] 2012 [citado el 16 de junio de 2.013]; 29(1)7-13. Disponible en: https://www.ncbi.nlm.nih. gov/pmc/articles/PMC5734839/pdf/JFOS-29-1-7.pdf.

11. Bass WM. Human osteology: a laboratory and field manual. 4o Ed. Missouri: Archeological society press; 1995. Capítulo 3, Postcranial skeleton; p 99-269.

12. Brothwell DR. Digging up bones. $3^{\circ}$ ed. New York: Cornell University Press; 1981. Chapter IV, Measurement and morphological analysis of human bones; p 100-2.

13. Cavalcanti A, Porto D, Maia A, Melo T. Estimativa da estatura utilizando a análise dentária: estudo comparativo entre o método de Carrea e o método modificado. Rev Odontol UNESP [Internet]. 2007 [citado el 05 de mayo de 2020]; 36(4):335-339. Disponible en: https:// www.revodontolunesp.com.br/article/588018107f8c9d0a098b4a51/pdf/rou-36-4-335.pdf

14. Rekhi A, Mohan C, Nagpal R, Singh S. Estimation of stature in a young adult indian population using the Carrea's index. J. Forensic Odontostomatol [Internet]. 2014 [citado el 05 de mayo de 2020]; 32(1):15-23. Disponible en: https://www.ncbi.nlm.nih.gov/pmc/articles/ PMC5734808/

15. Sruthi R, Sudhakara R, Nallakunta R, Jyothirmai K, Preethi M, Navya T. Carrea's Index and tooth dimensions - An Avant-Garde in stature estimation: An observational study. J Clin Diagn Res [Internet]. 2016 [citado el 04 de mayo de 2020]; 10(12):ZC33-ZC37. Disponible en: https://www.ncbi.nlm.nih.gov/pmc/articles/ PMC5296573/

16. Prieto L, Lamarca R, Casado A. La evaluación de la fiabilidad en las observaciones clínicas: el coeficiente de correlación intraclase. Medicina Clínica. [Consultado el 06 de mayo 2020]. Accesible en: https://www.mvclinic. es/wp-content/uploads/Prieto-Coeficiente-correlaci\%C3\%B3n-intraclase.pdf 
